\title{
YIPF2 is a novel Rab-GDF that enhances HCC malignant phenotypes by facilitating CD147 endocytic recycle
}

\author{
Shanshan Qi ${ }^{1}$, Linjia Su', Jing Li', Pu Zhao ${ }^{2}$, Qing Zhang ${ }^{3}$, Xiuran Niu' ${ }^{1}$ Jingyuan Liu', Guhe Jia', Xiaoxuan Wei',
} Jan Tavernier ${ }^{4}$, Jianli Jiang ${ }^{5}$, Zhinan Chen $^{5}$ and Sihe Zhang (10 ${ }^{1}$

\begin{abstract}
An increased surface level of CIE (clathrin-independent endocytosis) proteins is a new feature of malignant neoplasms. CD147 is a CIE glycoprotein highly up-regulated in hepatocellular carcinoma (HCC). The ability to sort out the early endosome and directly target the recycling pathway confers on CD147 a prolonged surface half-life. However, current knowledge on CD147 trafficking to and from the cell-surface is limited. In this study, an MSP (membrane and secreted protein)-cDNA library was screened against EpoR/LR-F3/CD147EP-expressed cells by MAPPIT (mammalian protein-protein interaction trap). CD147 co-expressing with the new binder was investigated by GEPIA (gene expression profiling interactive analysis). The endocytosis, ER-Golgi trafficking and recycling of CD147 were measured by confocal imaging, flow cytometry, and biotin-labeled chase assays, respectively. Rab GTPase activation was checked by GST-RBD pull-down and MMP activity was measured by gelatin zymography. HCC malignant phenotypes were determined by cell adhesion, proliferation, migration, Transwell motility, and invasion assays. An ER-Golgi-resident transmembrane protein YIPF2 was identified as an intracellular binder to CD147. YIPF2 correlated and co-expressed with CD147, which is a survival predictor for HCC patients. YIPF2 is critical for CD147 glycosylation and trafficking functions in HCC cells. YIPF2 acts as a Rab-GDF (GDI-displacement factor) regulating three independent trafficking steps. First, YIPF2 recruits and activates Rab5 and Rab22a GTPases to the endomembrane structures. Second, YIPF2 modulates the endocytic recycling of CD147 through distinctive regulation on Rab5 and Rab22a. Third, YIPF2 mediates the mature processing of CD147 via the ER-Golgi trafficking route. Decreased YIPF2 expression induced a CD147 efficient delivery to the cell-surface, promoted MMP secretion, and enhanced the adhesion, motility, migration, and invasion behaviors of HCC cells. Thus, YIPF2 is a new trafficking determinant essential for CD147 glycosylation and transport. Our findings revealed a novel YIPF2-controlled ER-Golgi trafficking signature that promotes CD147-medated malignant phenotypes in HCC.
\end{abstract}

\section{Introduction}

The aberrant expression and distribution of membrane antigens can create diverse malignant phenotypes in neoplasms ${ }^{1,2}$. Membrane antigens are processed in ER-

\footnotetext{
Correspondence: Sihe Zhang (sihezhang@nankai.edu.cn)

${ }^{1}$ Department of Cell Biology, School of Medicine, Nankai University, Tianjin,

China

${ }^{2}$ Department of Neurobiology, College of Life and Health Sciences,

Northeastern University, Shenyang, China

Full list of author information is available at the end of the article.

Edited by I. Amelio
}

Golgi biosynthetic network. Subsequently, they are delivered to cell-surface. ligand or antibody binding can activate or cluster membrane antigens, and stimulate their uptake through clathrin-mediated endocytosis (CME) or clathrin-independent endocytosis (CIE) $)^{3}$. Internalized antigens explore a series of sorting events. Then, they are either recycled back to cell-surface for another round of trafficking, or sorted to lysosomes or to proteasome for degradation, leading to irreversible termination of antigen-mediated functions ${ }^{4}$.

\section{(c) The Author(s) 2019}

(c) (i) Open Access This article is licensed under a Creative Commons Attribution 4.0 International License, which permits use, sharing, adaptation, distribution and reproduction cc) in any medium or format, as long as you give appropriate credit to the original author(s) and the source, provide a link to the Creative Commons license, and indicate if changes were made. The images or other third party material in this article are included in the article's Creative Commons license, unless indicated otherwise in a credit line to the material. If material is not included in the article's Creative Commons license and your intended use is not permitted by statutory regulation or exceeds the permitted use, you will need to obtain permission directly from the copyright holder. To view a copy of this license, visit http://creativecommons.org/licenses/by/4.0/. 
CD147 antigen is a type I transmembrane glycoprotein that belongs to immunoglobulin superfamily. Mature CD147 is an N-linked glycosylated protein and exists both in transmembrane and soluble forms in tumors ${ }^{5-8}$. Depending on the degree of glycosylation, CD147 is Western-blotted in two smear bands $(32-65 \mathrm{kDa})$, suggesting it exists in two forms: high-glycosylated CD147 (HG-CD147) and low-glycosylated CD147 (LG-CD147). Hepatocellular carcinoma (HCC) is one of the most lethal and prevalent cancers worldwide. We previously showed that CD147 is up-regulated in HCC, which promotes hepatocarcinogenesis, apoptosis inhibition, invasion, and distant metastasis ${ }^{9,10}$.

A complex regulation network controls CD147 synthesis and transport ${ }^{5-8}$. The endocytosis and recycling of CD147 have critical roles in tumor progression $^{11}$. Most studies revealed that CD147 is internalized through Rab5-activation-dependent CIE, which is associated with tubular endosomes and by-passes the merging with EEA1-positive endosomes ${ }^{12-15}$. Activation of Rab22a or Arf6 GTPases can further promote CD147trafficking, accelerating it entering the fast recycling pathway ${ }^{12,16,17}$. This might avoid the slow recycling pathway through Rab11-positive endosomes or the degradation pathway for CD147 $7^{15,18}$. It is evident that CD147 binds with different partners during its transport in cancer cells. For example, caveolin-1 associates with LG-CD147, restrict its biosynthetic conversion to HGCD147, and escorts LG-CD147 to cell surface ${ }^{19,20}$. Monocarboxylate transporters tightly associate with CD147 and influence its processing in Golgi as well as trans-localization to cell surface ${ }^{21,22}$. Cyp60 binding with CD147, assists CD147 transport from Golgi lumen to cell surface ${ }^{23,24}$. Other proteins, such as Gal-3 and Annexin A2, can also associate with CD147, affecting its clustering or trafficking in vesicles ${ }^{25-27}$. Although these proteins are involved in subcellular transport of CD147, none were found to be key regulator in its endocytic recycling process. In addition, previous identification of CD147 binders was performed in cell lysates or screened by yeast two-hybrid. That most likely missed the natural binders to CD147 during its trafficking.

In this study, we screened intracellular binders of CD147 using mammalian protein-protein interaction trap (MAPPIT) ${ }^{28}$. Interestingly, one of CD147 binders, YIPF2, was found to be a Rab-GDF recruiting and activating Rab5/Rab22a on ER-Golgi, promoting endocytosis, recycling, and secretion of CD147, and thus enhancing the malignant phenotypes of HCC cells. This benefits our understanding of the regulation mechanism on CD147 trafficking, and also supplies a target to disrupt the CD147 transport pathway in HCC progression.

\section{Results}

\section{YIPF2 is a new intracellular binder to CD147}

As CD147-stimulated MMP (matrix metalloproteinase) secretion involves its interaction with membrane and secreted protein (MSP) on neighboring cells ${ }^{5,7,8}$, we first established a human liver cancer-derived MSP-cDNA library (Supplementary Fig. 1). To screen the trafficking binders of CD147 under physiological condition, HEK293-16 cells expressing EpoR/LR-F3/CD147EF chimera receptor (as bait) were infected with retroviral MSPcDNA library (as prey) (Fig. 1a). After double selection with puromycin and Epo, eighteen clones that survived were screened. They contained six unique gene candidates (Supplementary Fig. 2a, b). Checking their function in UniProt database found that the yeast homolog of YIPF2 (Yif1p) is a Rab-bound protein involving vesicle transport (Table 1).

To further identify their interaction, extracellular and intracellular portions of CD147 (CD147EP and CD147IP) were included in analytical MAPPIT (Supplementary Fig. 2c), and specific binding of YIPF2 to CD147EP were detected (Fig. 1b). Confocal imaging of their distribution in HepG2 cells demonstrated that YIPF2 was co-localized with CD147 mainly at ER-Golgi network, sorting, and recycling endosomes (Fig. 2a, Supplementary Fig. 4). Furthermore, the co-localization between endogenous CD147/YIPF2 and ER marker KDEL, and Golgi markers GM130 (marking cis-Golgi) and TGN38 (marking transGolgi) were determined. Strong co-localized YIPF2CD147 was observed in ER and trans-Golgi network, whereas their co-localizations were not found in cis-Golgi, plasma membrane, or other membranous organelles (Figs 2b, 4a, Supplementary Figs. 4, 6, 7, 8b, and 9). In addition, co-IP results also validated the specific binding of YIPF2 with CD147 in cell lysates and membranous organelles (Fig. 2c-f). These results indicated that YIPF2 is a new binder to CD147, which is possibly involved in intracellular transport of CD147 via ER-Golgi biosynthetic network.

\section{Clinical significance of YIPF2-CD147 co-expressed in HCC}

To investigate the clinical significance of YIPF2-CD147 interaction, we next analyzed their expression by bioinformatics. Although CD147 and YIPF2 tend to be highexpressed in liver cancer patients, CD147 exhibited a higher transcription level in HCC tissues than in matched normal tissues (Fig. 3a). Moreover, both CD147 and YIPF2 expression were markedly up-regulated at stage IV of HCC (Fig. 3b). Survival analysis showed that a higher expression of CD147 was significantly associated with shorter disease-free survival (DFS) and overall survival (OS), whereas YIPF2 expression was slightly associated 


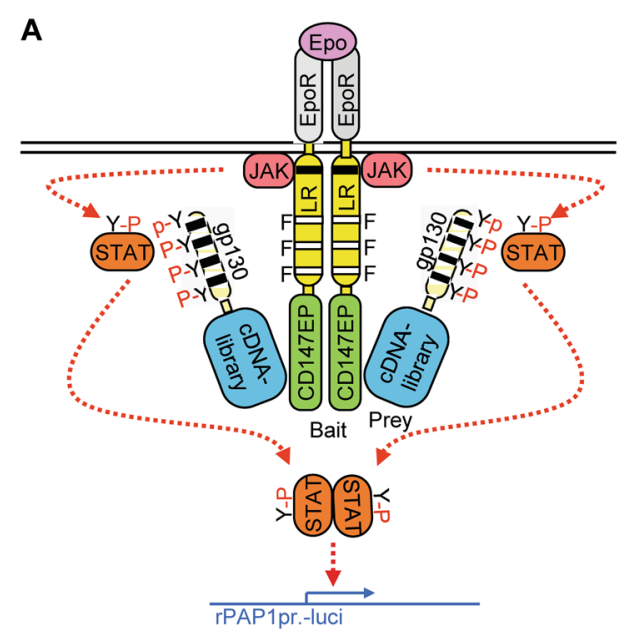

B

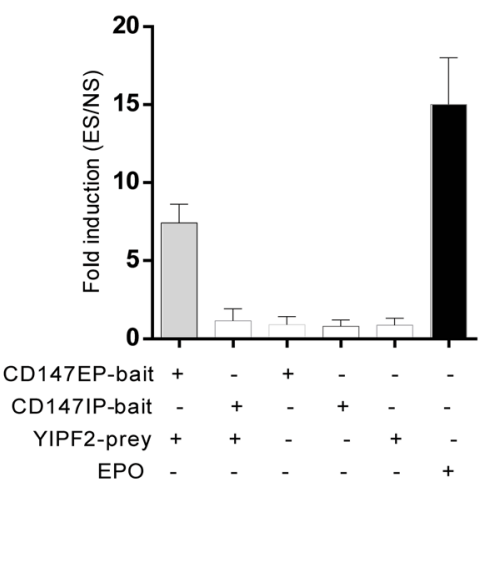

Fig. 1 YIPF2 is an intracellular binder to CD147 screened by MAPPIT. a Principle of screening MAPPIT. A heterologous bait CD147EP was fused to a chimera receptor consisting of the extracellular domain of homo-dimeric EpoR fused to the transmembrane and cytosolic domains of the LR-F3. Tyrosine to phenylalanine mutations (F3) eliminated the functional STAT3 recruitment sites at positions 1138, 985, and 1077 linking to the Ras pathway and to JAK-STAT signaling inhibitors. Heterologous prey polypeptides, i.e., MSP-cDNA library, were fused to the receptor fragment of gp130 containing four functional STAT3 recruitment sites. If bait and prey bound, the recruited receptor fragment was phosphorylated upon ligand-induced receptor activation, and a STAT3-dependent reporter gene signal was obtained. b Determining the binding of YIPF2 with CD147EP/IP by analytical MAPPIT. HEK293T cells were co-transfected with the pSEL1/CD147EP or pSEL1/CD147IP (bait) plasmid together with the pMG1/YIPF2 (prey) plasmid or a mock plasmid, and combined with the pXP2d2-rPAP1-luci plasmid. The transfected cells were stimulated for $24 \mathrm{~h}$ with EPO (ES) or were not stimulated (NS). Luciferase measurements were performed in triplicate, and positive interactions were considered significant if ES/NS $>5$

Table 1 MAPPIT-screened binder to CD147

\begin{tabular}{|c|c|c|c|c|}
\hline Clones & Protein & $\begin{array}{l}\text { Molecular } \\
\text { mass (kd) }\end{array}$ & Description & Molecular function (from UniProt) \\
\hline 1 & YIPF2 & 35 & Yip1 domain family,member 2 & Rab GTPase binding \\
\hline 2 & SCAP & 140 & $\begin{array}{l}\text { Sterol regulatory element-binding } \\
\text { protein cleavage-activating protein }\end{array}$ & $\begin{array}{l}\text { Escort protein required for cholesterol as well as lipid homeostasis. Regulates } \\
\text { export of the SCAP/SREBF complex from the ER upon low cholesterol }\end{array}$ \\
\hline 3 & MTG1 & 37 & $\begin{array}{l}\text { Mitochondrial ribosome-associated } \\
\text { GTPase } 1\end{array}$ & $\begin{array}{l}\text { Regulates the mitochondrial ribosome assembly and of translational activity. } \\
\text { Displays GTPase activity }\end{array}$ \\
\hline 4 & $\mathrm{CISH}$ & 29 & $\begin{array}{l}\text { Cytokine-inducible SH2-containing } \\
\text { protein }\end{array}$ & $\begin{array}{l}\text { Involved in the negative regulation of cytokines that signal through the JAK- } \\
\text { STAT5 pathway; protein kinase inhibitor activity }\end{array}$ \\
\hline 5 & PPCS & 34 & $\begin{array}{l}\text { Phosphopantothenoylcysteine } \\
\text { synthetase }\end{array}$ & $\begin{array}{l}\text { Catalyzes the first step in the biosynthesis of coenzyme A from vitamin B5. } \\
\text { phosphopantothenate-cysteine ligase activity }\end{array}$ \\
\hline 6 & NARF & 51 & Nuclear prelamin A recognition factor & Lamin binding; NADH dehydrogenase activity \\
\hline
\end{tabular}

with HCC survival (Fig. 3c, d). Interestingly, there were significant correlations between the expression of CD147 and YIPF2 both in HCC and in liver tissues $(p<0.01, R=$ $0.29-0.78$ ) (Fig. 3e). These results indicated that YIPF2 co-expressed with CD147 is a predictor for HCC survival. Further protein interactive network analysis retrieved a few sorting-associated proteins (e.g., ATP6V1F) for CD147. In contrast, quite a number of proteins, which involved in vesicular trafficking (e.g., TMED1, KDELR1) or ER-Golgi transport (e.g., COPE, ZFPL1, PRKCSH), were enriched for YIPF2 (Fig. 3f). These data suggested that YIPF2 possibly mediates the trafficking and/or maturation process of CD147.

As CD147 and MMP were highly expressed/secreted in HepG2 and 7721 cells (Supplementary Fig. 3), we focused on these two cells in the remained of this study.

\section{YIPF2 mediates the mature processing of CD147 in ER- Golgi network}

To ascertain whether YIPF2 functions in CD147 maturation process, YIPF2 stable knock-down (YIPF2KD) HepG2 cells were used to check CD147 retention in membranous organelles. Confocal imaging showed that 
A
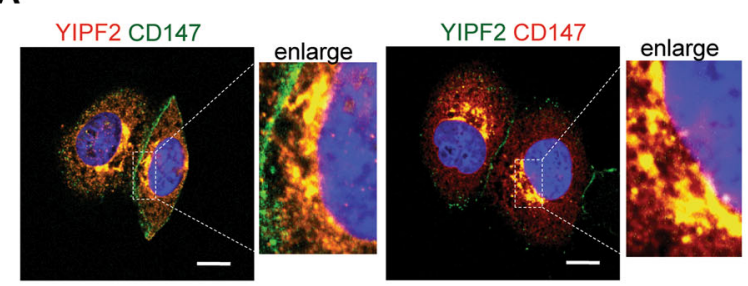

B
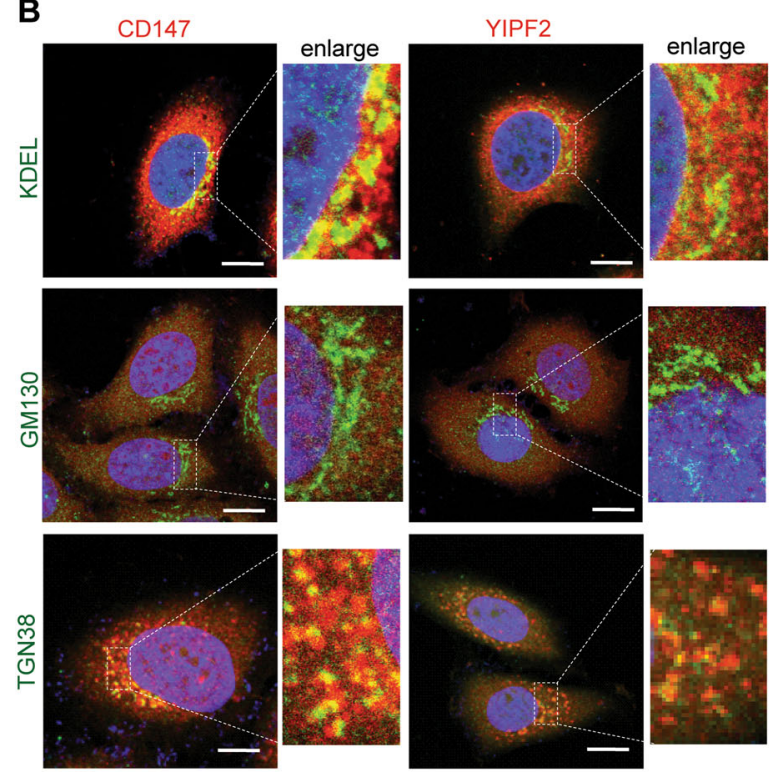

C

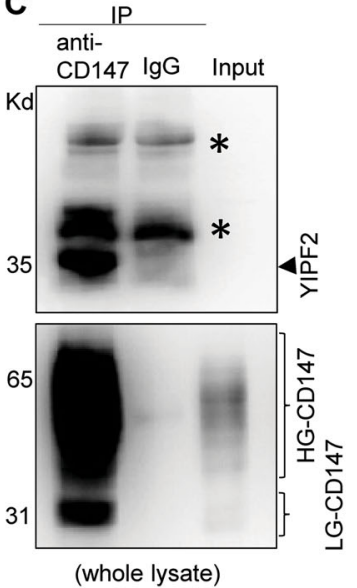

D

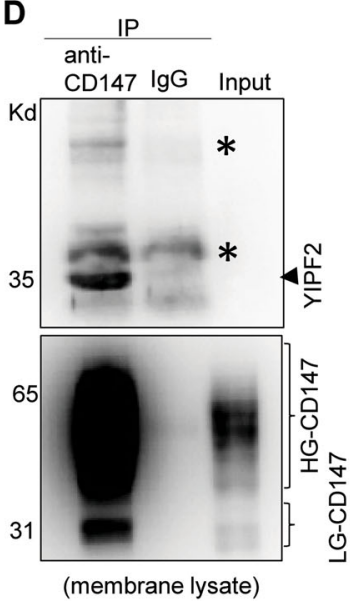

E

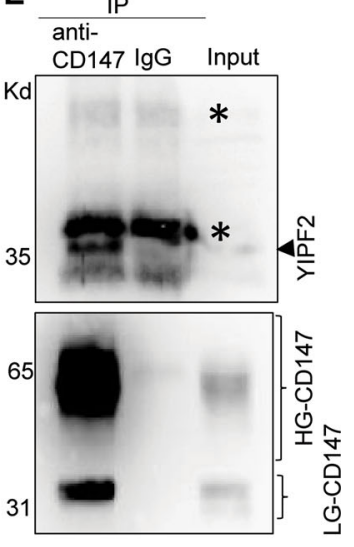

(ER lysate)

$\mathbf{F}$

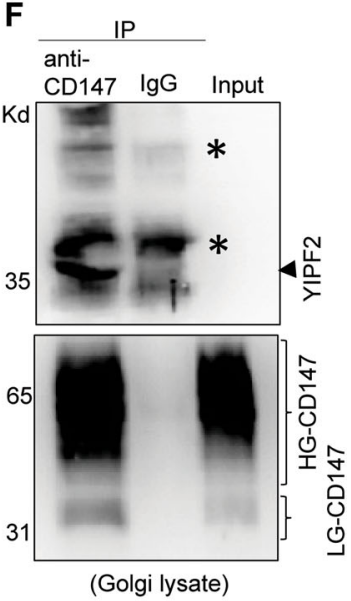

Fig. 2 YIPF2 co-localizes with CD147 at ER-Golgi organelles in HCC cells. a HepG2 cells were PFA-fixated, samponi-permeabilized, and stained with Ab combinations. Left: anti-YIPF2 Ab plus anti-rabbit Ab-AF594 (red color), H18Ab plus anti-mouse Ab-AF488 (green color). Right: anti-YIPF2 Ab plus anti-mouse Ab-AF488 (green color), anti-CD147 pcAb plus anti-rabbit Ab-AF594 (red color). b Confocal imaging of the organelle localization of YIPF2 and CD147. HepG2 cells were stained with Ab combinations: anti-YIPF2 Ab (red color, right panel) or anti-CD147 pcAb (red color, left panel) together with anti-KDEL Ab, anti-GM130 Ab and TGN38 Ab, respectively (both with anti-mouse Ab-AF488, green color). Representative observations are shown in (a-b). Scale bar: $20 \mu \mathrm{m}$. c-f co-IP analysis of the binding of YIPF2 with CD147. CD147 was immunoprecipitated from subcellular fractions (c whole lysate. $\mathbf{d}$ membrane lysate. e ER lysate. f Golgi lysate.) of HepG2 cells using H18 Ab, and co-immunoprecipitated YIPF2 were blotted with its $A b$

YIPF2-KD significantly dissipated ER- and Golgi-localized CD147, and such dissipation was rescued by YIPF2 overexpression (Fig. 4a, b; Supplementary Figs. 5a, 5b, 6). Notably, overexpression of other YIPF family members also rescued this dissipation in YIPF2-KD cells (Supplementary Figs. 5c, 7). YIPF2-KD induced CD147 dissipation on ER and Golgi implies that the glycosylation of CD147 might be affected. Therefore, we investigated the glycosylation level of CD147 in ER- and Golgi-fractions of HepG2 cells. Western blotting showed that both HGCD147 and LG-CD147 were markedly reduced in YIPF2KD HepG2 cells, and YIPF2 overexpression can rescue this reduction (Fig. 4c-f). Similar confocal and Western blot results were obtained in 7721 cells (Supplementary
Figs. 8, 9). These results suggested that YIPF2 can mediate the mature processing of CD147 via ER-Golgi trafficking route.

\section{YIPF2 controls the endocytosis and recycling of CD147}

Since abnormal glycosylation of proteins always arise from altered intracellular transport ${ }^{29-31}$, we then examined whether CD147 trafficking was affected by YIPF2 interference. Confocal imaging showed that YIPF2-KD significantly reduced CD147 uptake in HepG2 cells. Conversely, YIPF2 overexpression reversed the reduced uptake of CD147 in YIPF2-KD cells (Fig. 5a, b). This action of YIPF2 on CD147 uptake was confirmed by flow cytometry results (Fig. 5c). Coupled with these 

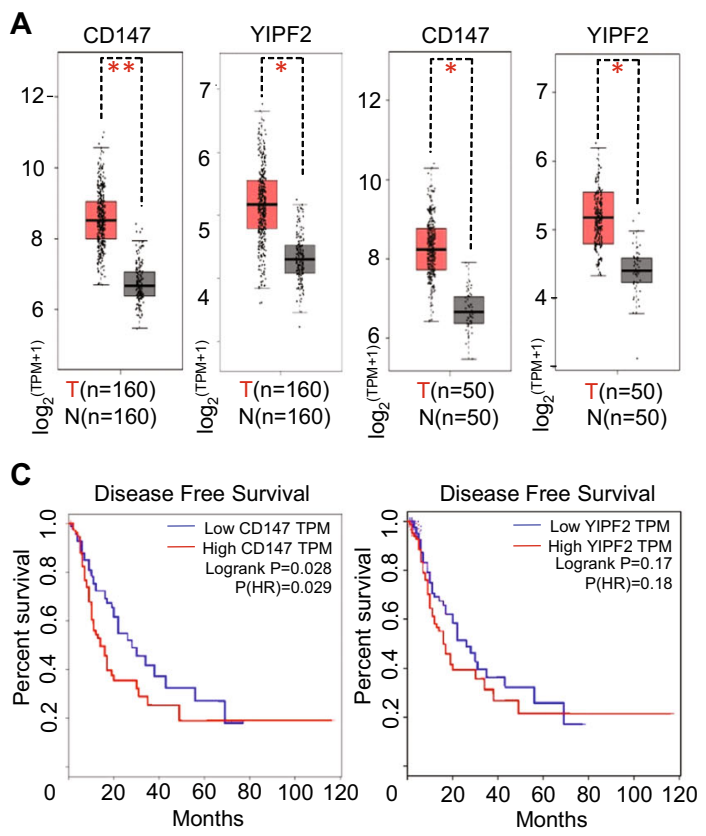

E
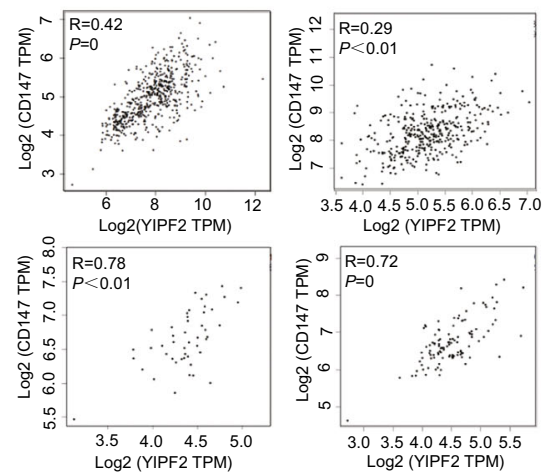

B

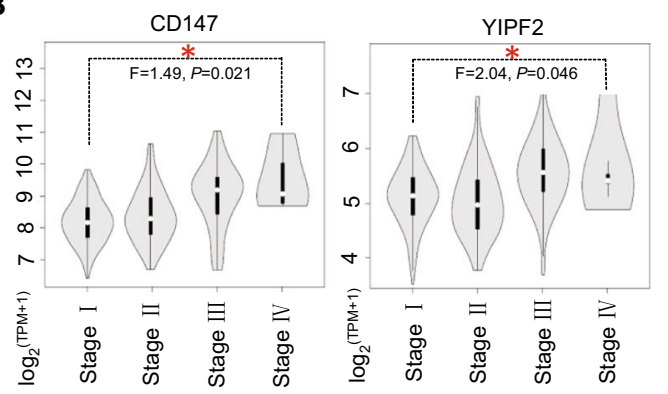

D
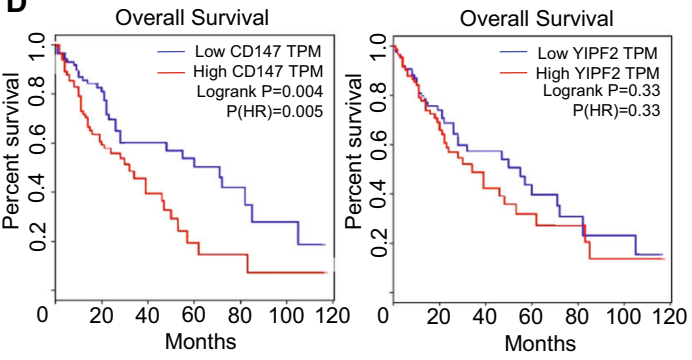

$\mathbf{F}$

\begin{tabular}{|c|c|c|c|}
\hline \multicolumn{2}{|c|}{ CD147 } & \multicolumn{2}{|c|}{ YIPF2 } \\
\hline Gene symbol & PCC & Gene symbol & PCC \\
\hline NDUFS7 & 0.54 & TMED1 & 0.61 \\
\hline COX4l1 & 0.49 & C19orf52 & 0.60 \\
\hline C19orf70 & 0.48 & PRKCSH & 0.56 \\
\hline CD320 & 0.48 & WDR830S & 0.56 \\
\hline TEX264 & 0.47 & KDELR1 & 0.55 \\
\hline ATP6V1F & 0.47 & SWSAP1 & 0.53 \\
\hline NDUFA13 & 0.47 & ELOF1 & 0.52 \\
\hline EMC10 & 0.46 & COPE & 0.51 \\
\hline NDUFA8 & 0.46 & ZFPL1 & 0.51 \\
\hline G6PC3 & 0.46 & FBXW9 & 0.42 \\
\hline
\end{tabular}

Fig. 3 Clinical significance of CD147 and YIPF2 co-expression in HCC. a Box plots depict the expression level difference between HCC samples (T) and normal tissues (N). Matched TCGA normal \& GTEx data (left two plots) and matched TCGA normal data (right two plots) are respectively shown. Each dot represents expression of a sample. Student's $t$-test, ${ }^{*} p<0.05,{ }^{* *} p<0.01$. b Stage plots depict the expression level variation among different pathological stages of HCC tissue samples. c Survival plots depict the disease-free survival (DFS) for low and high expression groups of the CD147 gene (left plots) and the YIPF2 gene (right plots) among HCC patients (cutoff: median). d Survival plots depict the overall survival (OS) for lo and, high expression groups of the CD147 gene (left plots) and the YIPF2 gene (right plots) among HCC patients (cutoff: median). e Pair-wise gene expression correlation analysis for the given sets of TCGA and/or GTEx expression data (left-upper: all mixed, right-upper: HCC tumor, left-below: HCCpaired normal tissues, right-below: liver). Pearson's $R$ test. $\mathbf{f}$ Co-expression network analysis of function-related genes was based on the datasets across all tumor samples and paired normal tissues. The lists show the top ten genes with functional connections

observations, Western blotting showed that YIPF2-KD increased membrane-resident CD147 level but decreased cytoplasmic CD147 pool in HepG2 cells (Fig. 5d-f). Similar results were obtained in 7721 cells (Supplementary Fig. 8a, c, d, j).

As bioinformatics analysis predicted YIPF2 being a Rab-bound protein, and Rab regulate the endocytic recycling pathway by different $\operatorname{modes}^{30,32}$, we next examined the recycling of CD147 after YIPF2 intervention. Confocal and flow cytometry results showed that YIPF2-KD significantly increased surface-resident CD147 pool, and the increment was eliminated by YIPF2 overexpression (Fig. 5g-i). Notably, co-IP checking the dynamic change of CD147 in HepG2 cells showed that YIPF2-KD markedly increased biotinlated CD147 on cell surface, and significantly accumulated biotinlated CD147 in cytosol fraction (residual pool after recycling) (Fig. 5j-1). Similar results were obtained in 7721 cells (Supplementary Fig. 8g, h, l). These data suggested that YIPF2 expression dictated the endocytic recycling of CD147 in HCC cells, implying its traffic-control function possibly through Rab GTPase regulation. 
A
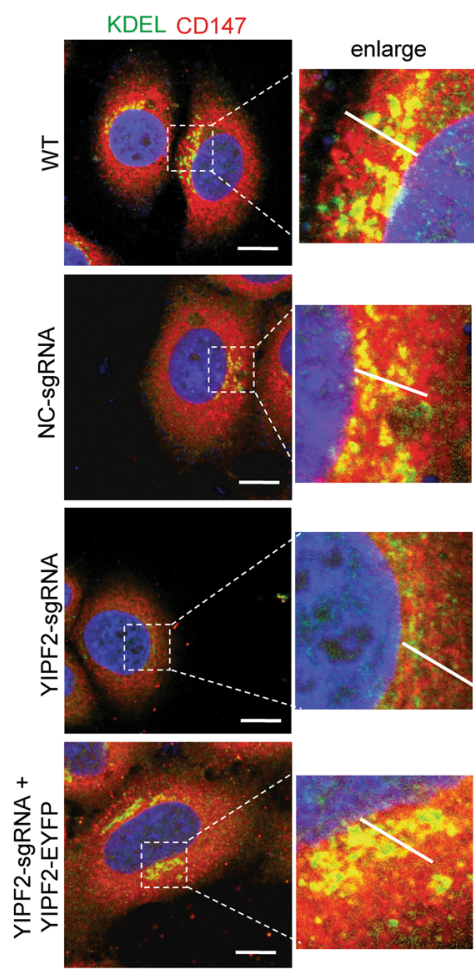

B

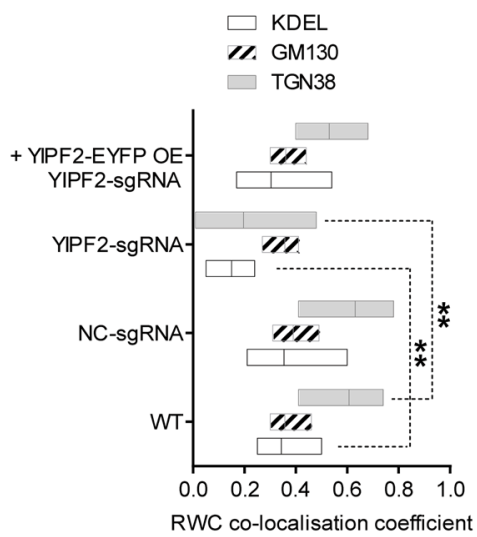

E

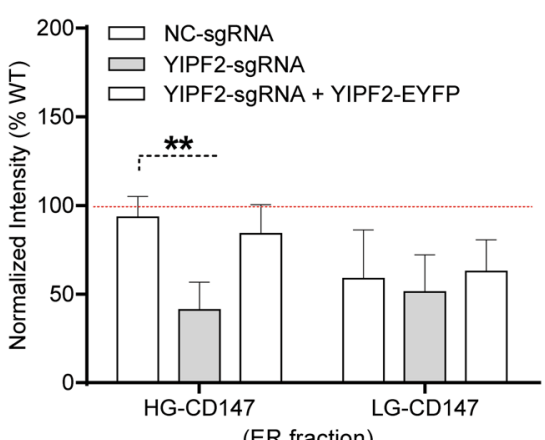

TGN38 CD147
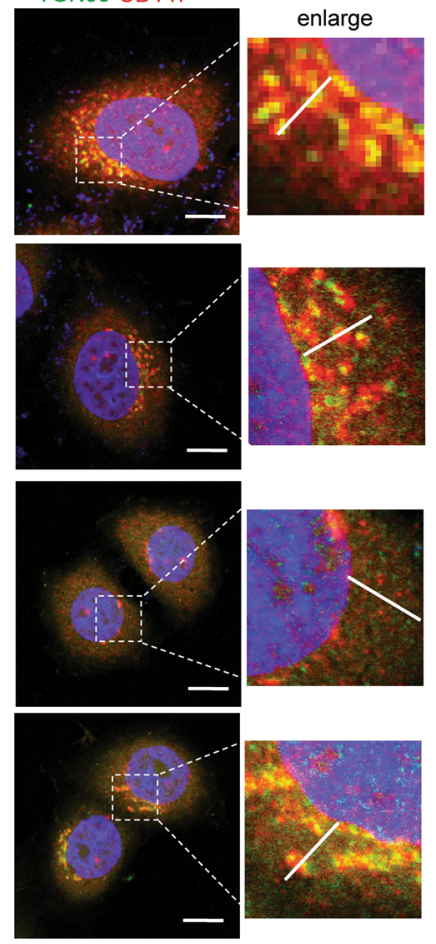

C

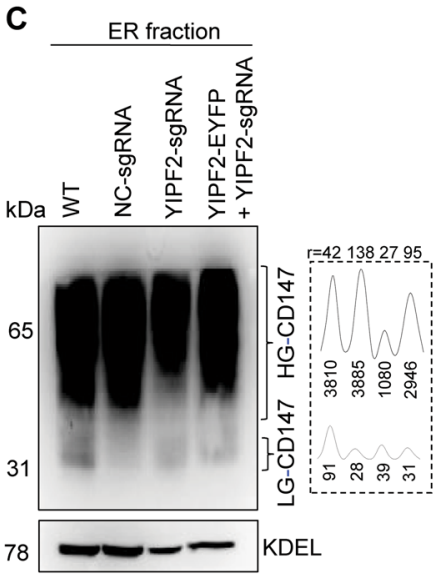

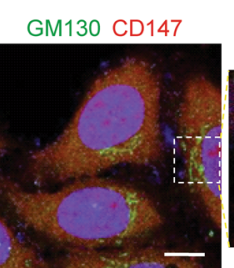

enlarge
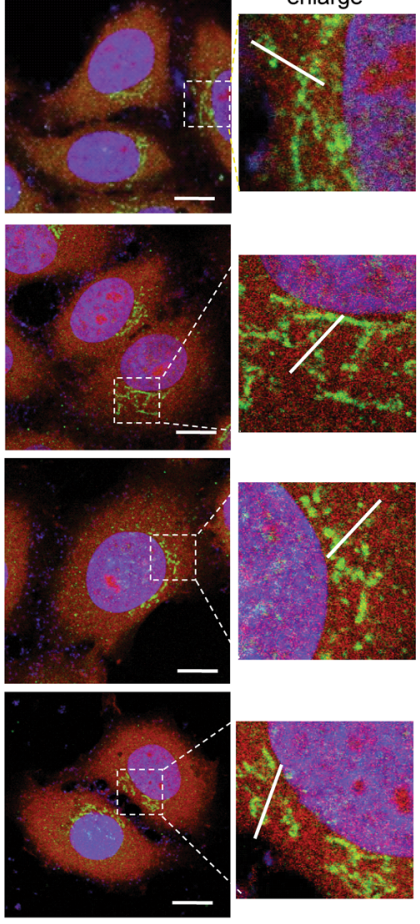

D
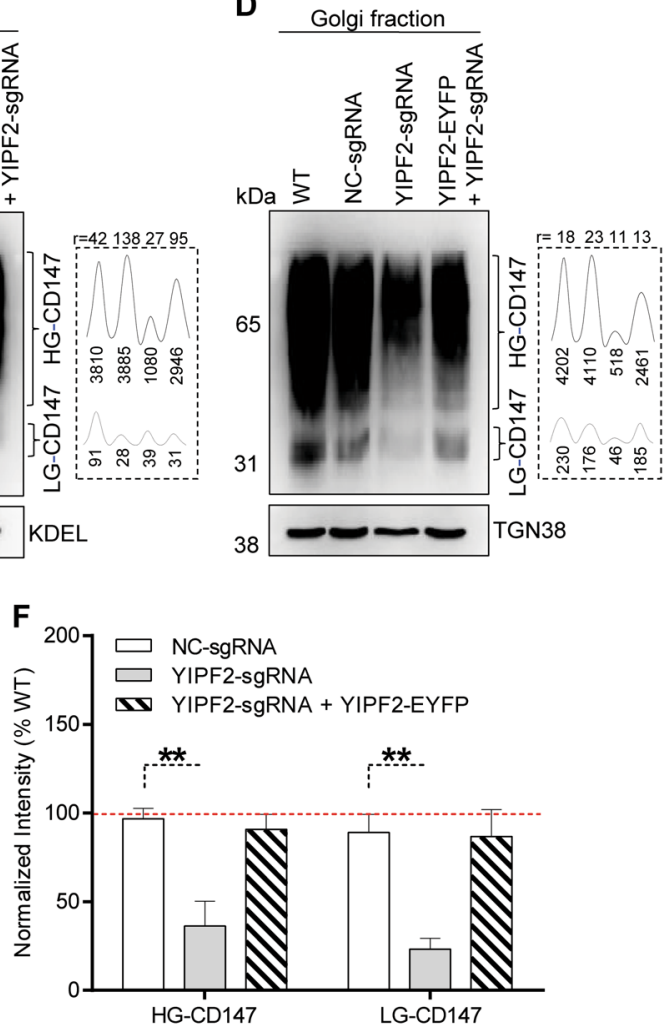

(Golgi fraction)

Fig. 4 (See legend on next page.) 
(see figure on previous page)

Fig. 4 YIPF2 mediates the maturation processing of CD147 via the ER-Golgi trafficking route. YIPF2 knock-down dissipated ER-/Gogli-localized CD147. YIPF2-KD HepG2 cells (NC-KD cells as controls. WT: non-transfected HepG2 cells) were PFA-fixed, samponi-permeabilized, and stained by Ab combinations: anti-CD147 pcAb together with anti-KDEL, anti-GM130 and anti-TGN38 Abs, respectively (both plus corresponding anti-rabbit/mouse Ab-fluorescence). a Representative confocal observations are shown in the merged model. Scale bar: 20 um. b Rank weighted coefficient (RWC) colocalization values of CD147 with KDEL, GM130, and TGN38. YIPF2 knock-down decreased the CD147 glycosylation level. Both HG- and LG-CD147 were blotted from the ER (c) and Golgi (d) fractions of treated HepG2 cells using H18 Ab. CD147 blotting (left) and quantitative scans of CD147 blots (right) by Image J software are shown. $r$ values refer to $\mathrm{HG} / \mathrm{LG}$ ratios. Numbers below indicate corresponding areas of CD147 peaks. Representative blot results from three independent experiments are shown $(\mathbf{c}, \mathbf{d})$, and corresponding quantitative data were analyzed (e, $\mathbf{f})$. Statistically significant differences compared with NC-KD cells are shown: $n=3,{ }^{* *} P<0.01$

YIPF2 activates and recruits Rab5/22a to ER-Golgi network

As Rab5 and Rab22a dominate the sorting and recycling of CIE proteins $^{30,31,33}$, we checked whether altered YIPF2 expression can affect their activation. GST pull-down results showed that YIPF2-KD significantly attenuated the activations of Rab5 and Rab22a, and such attenuation was rescued by YIPF2 overexpression in HepG2 cells (Fig. 6a-e). Given that Rab GTPases mediate protein transport highly relay on their tethering to membranous structures $^{29,34}$, we speculated that YIPF2-KD restrained the recruitment and activation of Rab5/Rab22a through ER-Golgi networks. Confocal imaging showed that YIPF2KD markedly weakened ER-localized Rab5wt and Rab5DA, whereas ER-localizations of wt-Rab22a and DARab22a were not affected in HepG2 cells (Fig. 6i, j. Supplementary Fig. 10). In contrast, YIPF2-KD only weakened the localizations of wt-Rab22a and DA-Rab22a in Golgi (Fig. 6i-k. Supplementary Fig. 10). Further determining the membrane-associated Rab showed that YIPF2-KD caused Rab22a activation to decrease in all checked membranous fractions (Fig. 6b-h). However, no Rab5 activation reduced in Golgi was detected after YIPF2-KD (Fig. 6d-h). Together, these data suggested that Rab5/22a recruitment and activation at ER-Golgi network depend on YIPF2 protein level.

\section{YIPF2 modulates CD147-mediated malignant phenotypes}

MMP-mediated extracellular matrix (ECM) degradation is essential to HCC invasion, and MMP secretion is trigged by CD147-mediated intercellular interaction ${ }^{5-8}$. Gelatin zymography results showed that YIPF2-KD significantly increased MMP2 and MMP9 secretion in HepG2 cells, but such an increase was rescued by YIPF2 overexpression (Fig. 7a-c). Although YIPF2-KD induced MMP secretion increase were not detected in co-culture medium (Fig. $7 \mathrm{~b}-\mathrm{d}$ ), the promotion phenomena reappeared when HepG2 medium was added to 3T3 cells (Fig. 7e-g). Notably, when CD147 was depleted from cell medium, YIPF2-KD or overexpression induced MMP2/ 9 secretion from 3T3 cells were attenuated (Fig. 7e-g). Additionally, Western blotting showed that YIPF2-KD significantly reduced endogenous MMP2 and MMP9 levels in HepG2 cells, and such a reduction was rescued by YIPF2 overexpression (Fig. 7f-h). Similar results were obtained in 7721 cells (Supplementary Fig. 11). These results suggested that YIPF2-KD induced CD147 delivery to HCC cell-surface and release to extracellular medium, which promoted MMP secretion.

As YIPF2 is critical for CD147 trafficking, we further speculated that YIPF2-KD highly induced CD147 presenting on cell-surface and more extensively promoted the malignant phenotypes of HCC cells. Our results showed that YIPF2-KD markedly increased the adhesion of HepG2 and 7721 cells to Matrigel, but this potential was reversed by YIPF2 overexpression (Fig. 8a). Since altered ECM adhesion and MMPs secretion are correlated with modified motility, migration, and invasion capabilities of cancer cells ${ }^{32}$, we then checked these behaviors. As expected, YIPF2-KD markedly enhanced the motility, migration, and invasion of HepG2 and 7721 cells. Conversely, YIPF2 overexpression remarkably restrained these characteristics (Fig. 8c-h). We also checked the contribution of cell proliferation to these characteristics, and no cell proliferation was affected by YIPF2 intervention (Fig. 8b). Together, these results suggested that YIPF2-KD enhanced the adhesion, motility, migration, and invasion behaviors of HCC cells.

\section{Discussion}

The function of CD147 as a driver of malignant neoplasms strongly depends on its cell surface presentation. Although several binders were reported to form complexes with $\mathrm{CD} 147^{19-27,35,36}$, none appear to govern CD147 trafficking in cancer cells. MAPPIT offers the combined advantages of optimal physiological context, separated interactor-effector design, and stimulationresponsive screening to determine dynamic interactions during antigen trafficking ${ }^{28,37}$. We used the advantages of MAPPIT, screened and identified YIPF2 as a new binder to CD147 with endocytic recycling regulation function.

The name of YIPF refers to the Ypt (yeast Rab GTPase)-interacting protein. Previous studies showed that YIPF proteins have five transmembrane segments with $\mathrm{N}$-terminal exposed to the cytoplasm and $\mathrm{C}$ - 


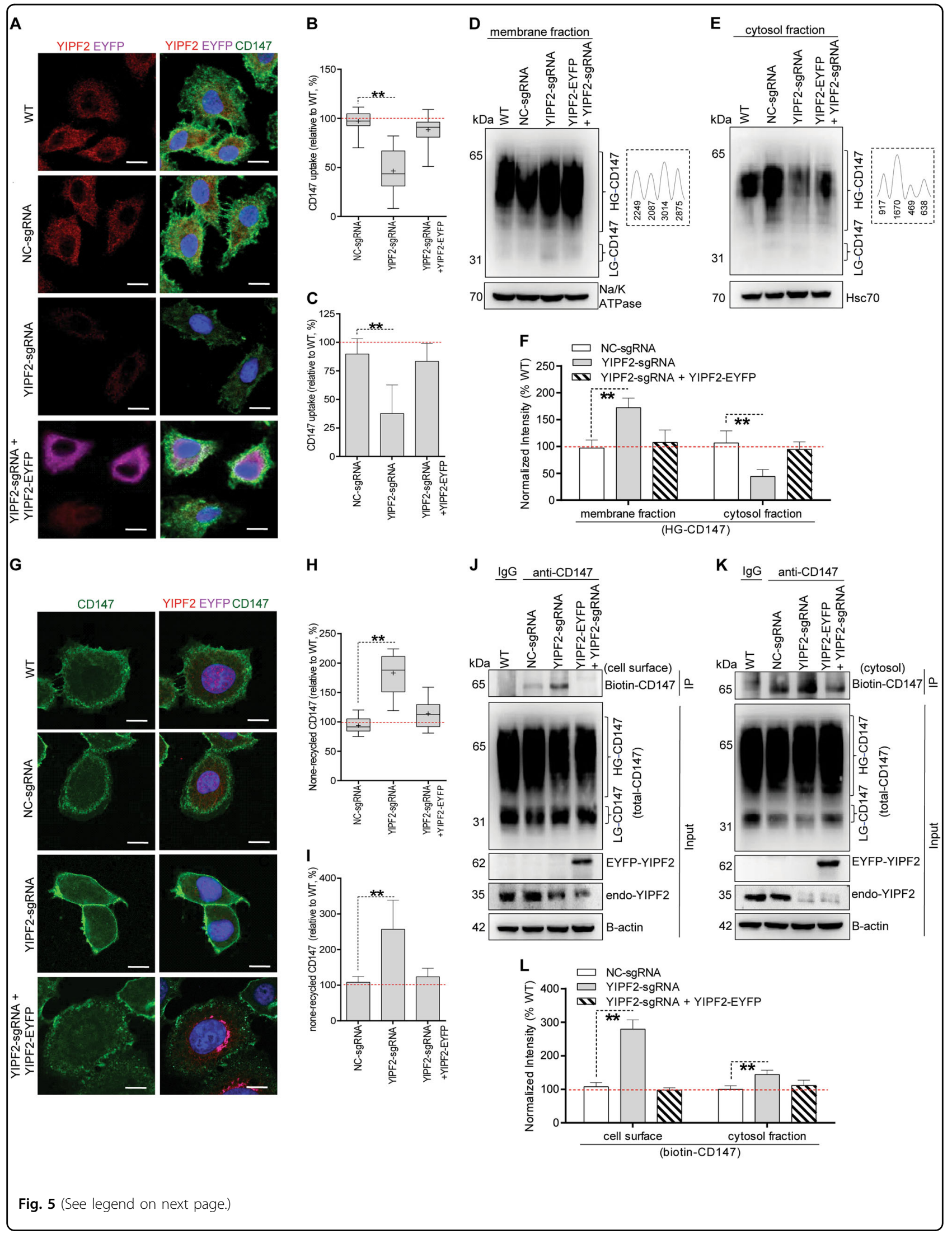


(see figure on previous page)

Fig. 5 YIPF2 controls the endocytosis and recycling of CD147. YIPF2 knock-down reduced CD147 uptake. YIPF2-KD HepG2 cells (NC-KD cells as control) were transfected with the YIPF2/pdEYFP plasmid, then incubated with the H18Ab-AF488 complex at $37^{\circ} \mathrm{C}$ for uptake, quickly rinsed, fixed, and Ab-stained (anti-YIPF2 to stain endogenous protein, anti-GFP to stain exogenous protein), and visualized via a confocal microscope. a Representative observations are shown. Scale bar: $20 \mu \mathrm{m}$. b Box-and-whiskers plots depict the uptake of the H18Ab-AF488 complex in cell populations. $n=60$. c After cell surface digestion, the uptake of the H18Ab-AF488 complex in cells was quantified by flow cytometry. YIPF2 knockdown impaired glycosylated CD147 trafficking. HG-CD147 were blotted from the membrane (d) and the cytosol fraction (e) of treated HepG2 cells using H18 Ab. Quantitative scanning of CD147 blots are shown in the right dotted line box. YIPF2 knock-down decreased CD147 recycling. After removing the surface-bound $\mathrm{H} 18 \mathrm{Ab}-\mathrm{AF} 488$ complex, cells were removed at $37^{\circ} \mathrm{C}$ for $30 \mathrm{~min}$ and the recycled complex on the cell surface was again removed. The no-recycling complex (i.e., intracellular residual portion) was visualized via a confocal microscope. $\mathbf{g}$ Representative observations are shown. Scale bar: $20 \mu \mathrm{m}$. h Box-and-whiskers plots depict the no-recycled CD147 pool in different cell populations. i The no-recycling H18Ab-AF488 complex inside HepG2 cells was quantified by flow cytometry. The uptake or no-recycled CD147 in untreated cells (WT) was set as 100\% for comparison. Significant differences compared with NC-KD cells are shown: $n=3,{ }^{* *} p<0.01$. Western blot determined the surface-resident biontinCD147 (j) and intracellular non-recycled biotin-CD147 (k) after cell-surface biotinylation and anti-CD147 immunoprecipitation as described in 'Materials and methods'. Representative blot results from three independent experiments are shown $(\mathbf{d}, \mathbf{e}, \mathbf{j}, \mathbf{k})$, protein bands were quantified by Image J software, and corresponding quantitative data were analyzed ( $\mathbf{f}, \mathbf{I})$. Statistically significant differences compared with NC-KD cells are shown: $n=3,{ }^{* *} p<0.01$

terminal exposed to the lumen of secretory endomembranes $^{38}$. Because YIPF proteins form complexes with each other and are found in ER, Golgi, and COPII vesicles, they were predicted to play roles in maintaining ER-Golgi morphology and/or in vesicle transport between organelles ${ }^{38-40}$. We found here that YIPF2 is mainly localized in ER and trans-Golgi of HCC cells (Fig. 2b). Unlike wide localization across endomembrane in YIPF2-overexpressed cells ${ }^{38}$, we hardly viewed endosome-localized YIPF2 in HCC cells unless Rab5/22 was also stained for co-localization analysis (Supplementary Fig. 4b). We found that YIPF2-KD dissipated ER- and trans-Golgi-localized CD147, and these dissipations coincided with ER-Golgi fragmentation and dispersion in HCC cells (Fig. 4a, b). This result is in contrasted with a recent study that YIPF2-KD induced no Golgi structure alteration but delayed Golgi reassembly in colon cancer cells ${ }^{41}$. The reason why YIPF3 and YIPF4 overexpression could rescue the dissipated ER-Golgi-localized CD147 in YIPF2-KD cells is not known (Supplementary Fig. 7). Although YIPF3 and YIPF4 are cis-Golgi-localized YIPFs, they always act together with trans-Golgi-localized YIPF2 on regulating endomembrane dynamics. As previous studies showed YIPFs co-precipitates with the components of cellular trafficking machineries and synergistically play roles in cargo trafficking ${ }^{39,42}$, it is not surprising that YIPF3/4 overexpression recovered both impaired Golgi morphology and distributed secretory pathway in HCC cells.

Being an $\mathrm{N}$-linked glycosylated protein, immature CD147 is preliminary glycosylated with 1 glycan in ER, and then transported to Golgi for further modification by glycosyltransferases including GnT-III, GnT-IV, GnT-V, and FuT $-8^{6}$. As glycosyltransferases cannot work without integrated ER/Golgi morphology and YIPF2 interference hinders intracellular glycan synthesis ${ }^{41}$, it is logical that LG- and HG-CD147 are reduced in ER-Golgi fractions from YIPF2-KD HCC cells (Fig. 4c-f). Some researchers may argue that Golgi is the apparatus wherein O-linked glycan chains are synthesized, how could the N-linked glycosylated CD147 be affected by YIPF2-induced Golgi morphology change? One explanation is that $\mathrm{N}$ glycosylation modifications occur in both ER and Golgi. Another may be because Yif1p associates with Yip1p (yeast homolog of YIPF6) and plays role in membrane fusion within Golgi $^{43}$. Considering that branched Nglycans are catalyzed by GnT family glycosyltransferases which mainly located in Golgi ${ }^{6}$, and CD147 is a target of $\mathrm{GnT}^{-} \mathrm{V}^{44}$, it is probable that YIPF2-KD attenuated YIPF2-YIPF6 complex, causing delayed recycle of glycosyltransferases or incomplete vesicle fusion in Golgi, which impaired the glycosylation maturation of CD147 in ER-Golgi compartments (Fig. 9).

Rab GTPases regulate the trafficking of membrane proteins $^{30,31,34}$. Previous studies showed Rab5 activation boosted the early steps of CD147 uptake, and Rab22a activation accelerated CD147 recycling to cell surface ${ }^{33,45}$. We found that Rab5 and Rab22a activation were reduced by YIPF2-KD in HCC cells, and such reductions were severe in membrane fractions (Fig. 6a-f). This result is logical, because the yeast Yif1P is Rab-bound protein, and $\mathrm{Rab}$ is activated only when it was periodically recruited to membranes $^{46}$. One interesting finding is that YIPF2-KD induced distinct inactivation and dissipation of Rab GTPases (Fig. 6). Although Rab5 can work together with other GTPases to direct CIE cargos (such as CD147) transport to recycling endosomes, Rab22a is key driver for CIE sorting machinery ${ }^{33}$. The more inactivation of Rab22a than Rab5 by YIPF2-KD (Fig. 6a-h), the more recycling of CD147 was impaired, suggesting YIPF2 mainly functions on the late sorting steps of CD147.

What is the role played by YIPF2 on CD147 transport? Because yeast YIP1 family are counterparts to Rab-GDI (guanine nucleotide dissociation inhibitor) and they 

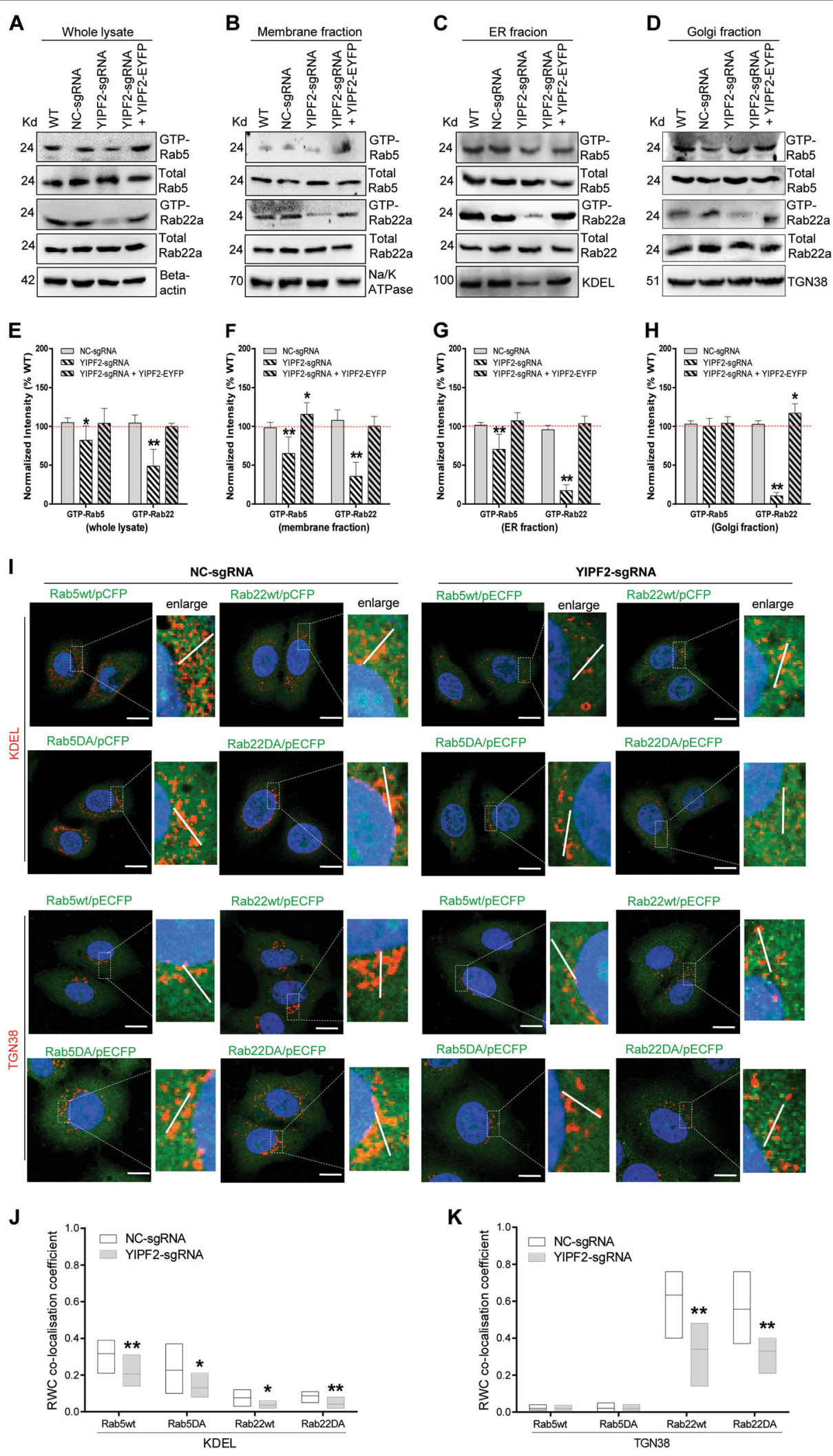

Fig. 6 (See legend on next page.) 


\begin{abstract}
(see figure on previous page)
Fig. 6 YIPF2 activates and recruits Rab5/22a to the ER-Golgi network. a-d YIPF2 knock-down decreased the activation of Rab5/22a. YIPF2-KD HepG2 cells (NC-KD cells as controls) when transfected with the YIPF2/pdEYFP plasmid and continued growth for $32 \mathrm{~h}$. The cells were then serumstarved, lysed to extract different fractions, and incubated with GST-EEA or -Rabenosyn-5 beads. The activated Rab GTPases in the pull-down precipitate were blotted with corresponding Abs. Representative blot results from three independent experiments are shown. Activation of Rab GTPases was quantified by Image J software and shown in (e-h). Statistically significant differences compared with un-transfected cells (wt) are shown: $n=3,{ }^{* *} p<0.01,{ }^{*} p<0.05$. i YIPF2 knock-down dissipated the ER-/Gogli-localized Rab5/22a. Confocal imaging of the co-localization of wt- or DA-Rab5/22a with the ER marker (KDEL) and Golgi marker (TGN38). HepG2 cells were PFA-fixed, samponi-permeabilized, and stained with different Ab combinations: anti-GFP pcAb (plus anti-rabbit-AF488, green color) together with anti-KDEL Ab or anti-TGN38 Ab (both plus anti-mouse Ab-Alexa Fluor594, red color). Representative observations are shown. Scale bar: 20 um. j, k Box-and-whiskers plots depict the co-localization variation of Rab5/ 22a with KDEL or TGN38 in cell populations. The rank weighted coefficient (RWC) values were quantified as described in 'Materials and methods'. Significant differences compared with NC-sgRNA transfection are shown: $n=3,{ }^{* *} p<0.01,{ }^{*} p<0.05$
\end{abstract}

complex together to establishing the fusion of ER/Golgi vesicles at the time of budding ${ }^{40,46}$, the best possibility is that YIPF2 serves as an acceptor which aids in recruiting Rab from the cytosol onto endosomes or ER-Golgi, enabling Rab to be correctly localized, activated, and used for many rounds of transport. Although we did not check whether YIPF2 affects other Rab GTPases, one study showed that Yiflp can bind with several Rab GTPases ${ }^{46}$. Notably, these Yif1p-bound Rabs are required for vesicle transport in endocytic pathway (YPT10, YPT52), secretory pathway (YPT31, YPT32, SEC4), endosome docking or fusion (VPS21, YPT7), ER-Golgi trafficking (YPT1), and post-Golgi secretion to cell surface (YPT6, SEC4) ${ }^{46}$. Because Rab5 and Rab22a activation trigger CD147 endocytic recycling ${ }^{33,45,47}$ and YIPF2 determines their activation and localization (Fig. 6), we propose a model: Rab5 and Rab22a bind to GDI in cytosol, which keeps them in inactive state. YIPF2 acts as a GDF (GDI-displacement factor) to catalyze the dissociation of Rab-GDI complexes, and to enable transfer of Rab5/22a from GDI onto distinct endosomes or ER-Golgi membranes. Activated Rab5 and Rab22a localize to distinct compartments and regulate CD147 transport from endocytosis, sorting and recycling to ER-Golgi trafficing/secretion (Fig. 9).

In summary, we identified YIPF2 as a Rab-GDF regulating CD147 endocytic recycling, and the surface level of CD147 is controlled by YIPF2 in HCC cells. Our results reveal a YIPF2-controlled ER-Golgi trafficking signature promotes CD147-medated malignant phenotype in HCC.

\section{Materials and methods}

\section{Cell cultures, plasmids, antibodies, and chemicals}

Materials are listed in Supplementary 'detailed material and methods'.

\section{Construction of retroviral MSP-cDNA library}

The process was performed as previously described ${ }^{10}$. Briefly, total RNA was drawn from two human liver cancer tissues. The MSP-enriched cDNA gene pool was PCR-amplified by using primers (Supplementary Table 1), digested with EcoR I/Not I, and cloned into pBG1 plasmid. The recombinant plasmids were electro-transformed into E. coli JM109 cells and culture-amplified. MSPcDNA library plasmids were transfected into Plat-E cells. One flask of cells was transfected with retroviral cDNA library and EGFP reporter plasmid. The culture supernatant containing retroviruses was harvested; viral morphology and titer were determined by electron microscopy and flow cytometry, respectively.

\section{MAPPIT screening and analysis}

MAPPIT were performed as previously described ${ }^{28}$. Briefly, EpoR/LR-F3/CD147EP-expressed HEK293-16 cells $\left(1.5 \times 10^{6}\right)$ were cultured in medium containing Epo $(5 \mathrm{ng} / \mathrm{ml})$ with puromycin $(0.5,1,1.5$, and $2 \mu \mathrm{g} / \mathrm{ml})$. The background was determined based on the number of colonies ( 1 or 2 per flask) that survived under doubleselection, but without infection by the retroviral cDNA library. chimeric receptor-expressed Cells were infected with the retroviral MSP-cDNA library, and then cultured under double selection. Total RNA was drawn from the surviving colonies, and genes of potential binders were PCR-amplified and cloned into pMD18-T vector. For analytical assays, HEK293T cells $\left(3 \times 10^{5}\right)$ were cotransfected with bait plasmid (pSEL1/CD147EP or pSEL1/CD147IP) and prey plasmid (pMG1/YIPF2) together with reporter (pXP2d2-rPAP1-luci) plasmid. After overnight growth, cells were reseeded in plates for EPO $(5 \mathrm{ng} / \mathrm{mL}, 24 \mathrm{~h})$ stimulation or not. Survived cells were lysed followed by adding luciferase substrate for activity analysis.

\section{Gene stable knock-down and transient overexpression}

The CRISPR/Cas9 lentivirus system (from Addgene) was utilized to produce YIPF2-KD HCC cells. Three independent YIPF2-targeting sgRNA (sgRNA\#1: CACCGCGAGGAGGCCACTAATCTTC, sgRNA\#2: CACCGGACCTTCCATGAATTCGAGG, and sgRNA\#3: CACCGTAGTGGCCTCCTCGAATTCA) were generated as previously described ${ }^{48}$. YIPF2/3/4pdEYFP and 

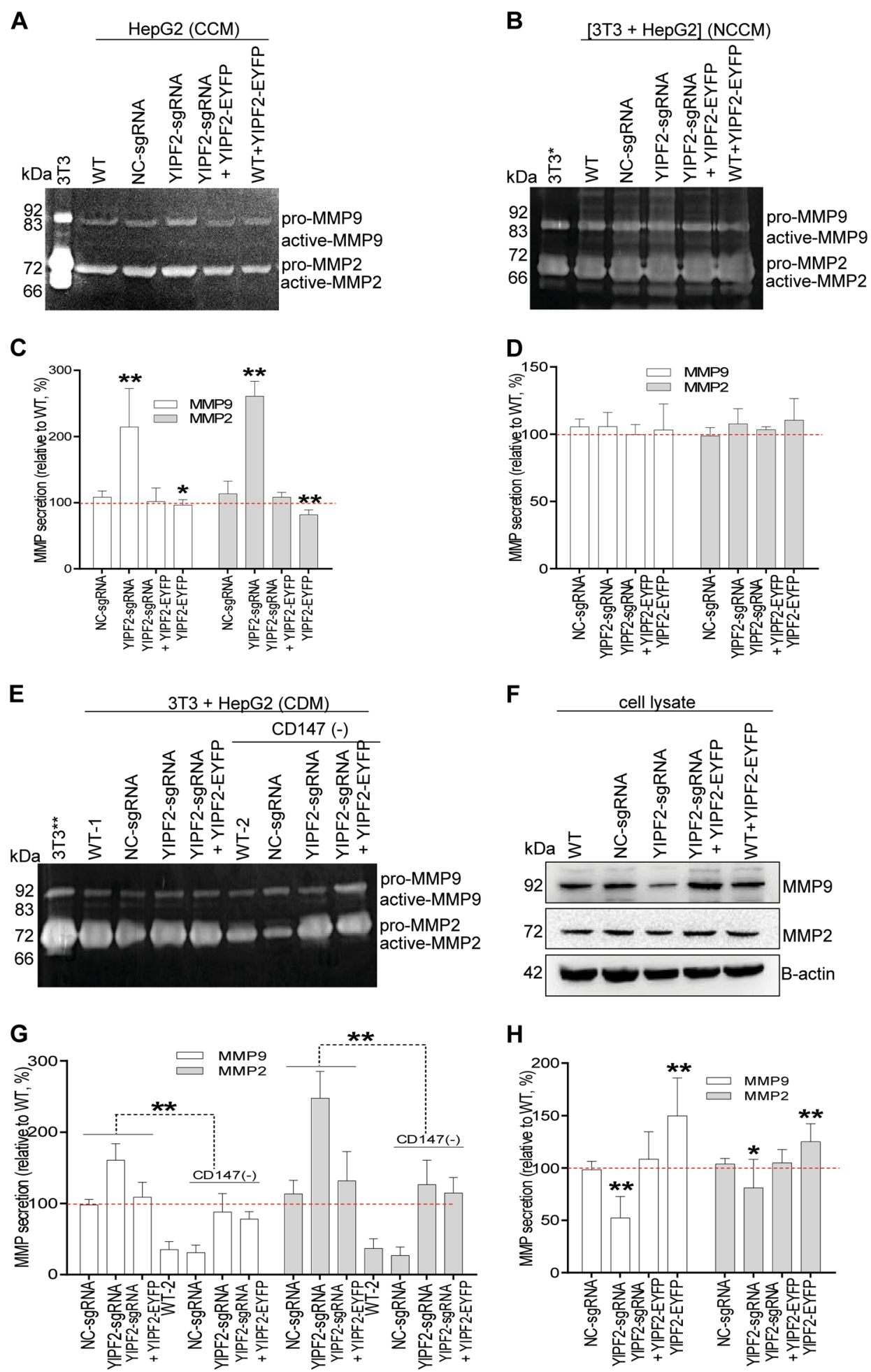

H

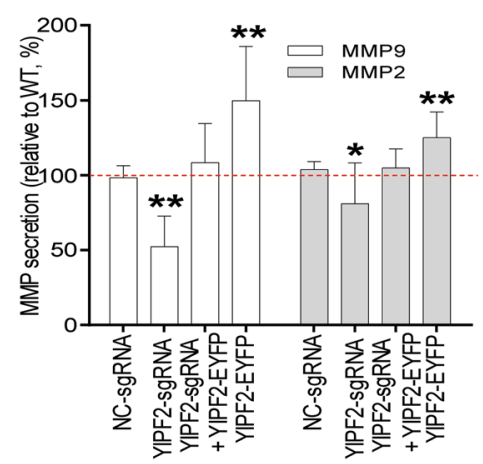

Fig. 7 YIPF2 knock-down increased MMP secretion. YIPF2-KD HepG2 cells were transfected with the YIPF2/pEYFP plasmid. a, b, e Forty-eight hours after transfection, secreted MMPs activity in concentrated medium (CCM) from mono-culture (a), in non-concentrated medium (NCCM) from co-culture with $3 T 3$ cells (b), and in conditioned medium (CDM, pre-depleted CD147 by H18 Ab) from 3T3 cells (e) were detected by gelatin zymography. $\mathbf{f}$ Western blot determined the endogenous MMP in treated cells. Representative results from three independent experiments are shown $(\mathbf{a}, \mathbf{b}, \mathbf{e}, \mathbf{f})$, protein bands were quantified by Image J software, and corresponding quantitative data were analyzed $(\mathbf{c}, \mathbf{d}, \mathbf{g}, \mathbf{h})$. Statistically significant differences compared with non-treated cells (WT) are shown: $n=3,{ }^{* *} p<0.01,{ }^{*} p<0.05$ 

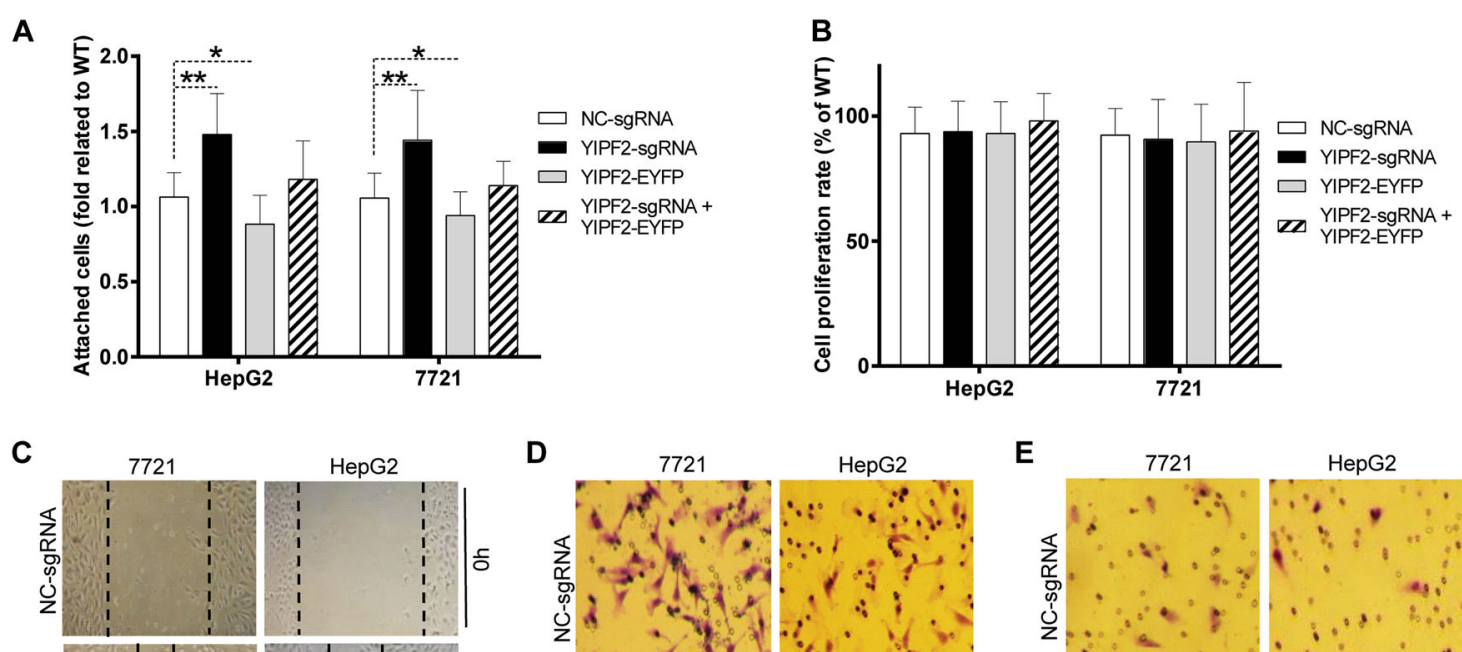

D

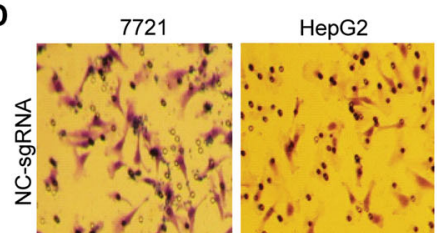

E
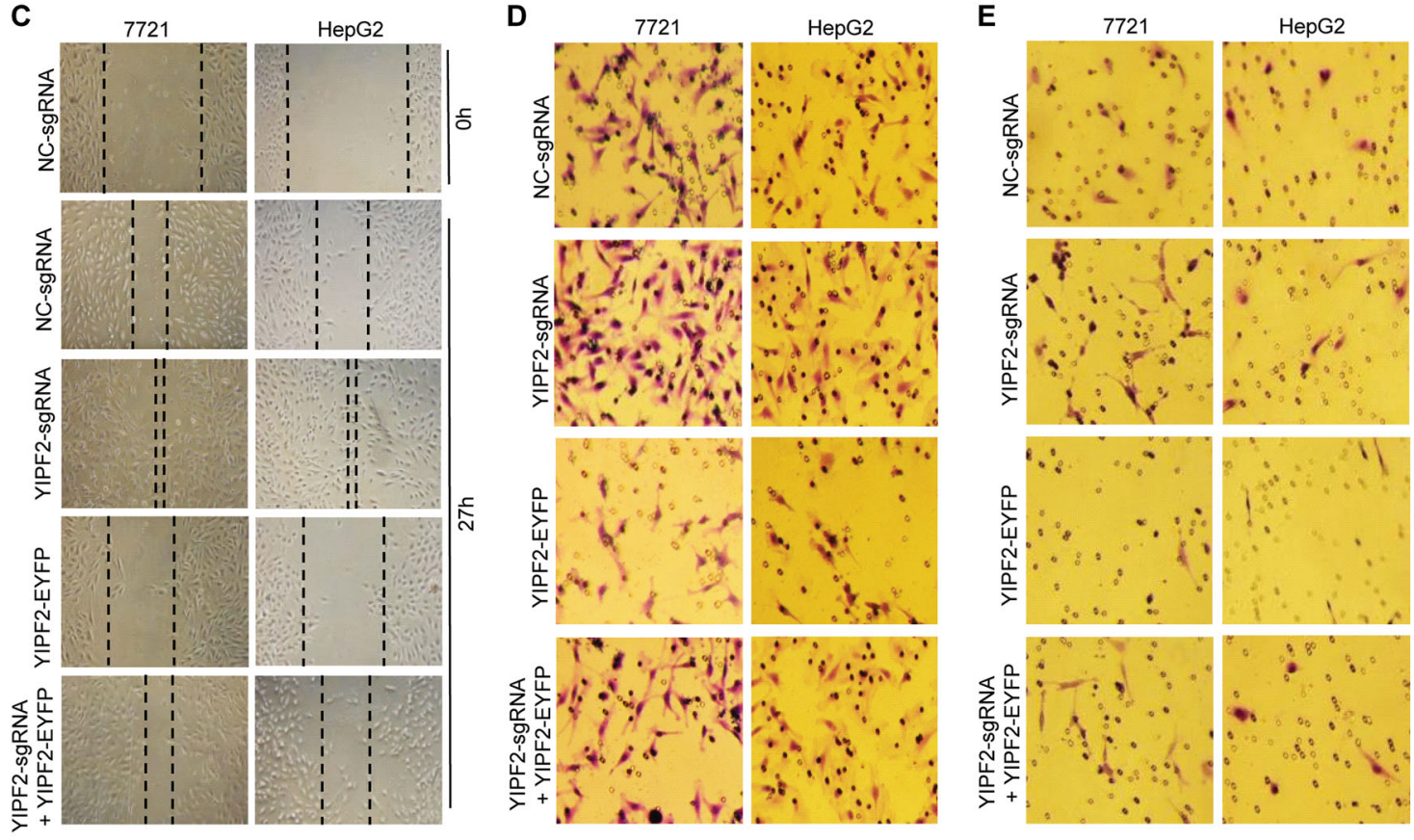

$\mathbf{F}$
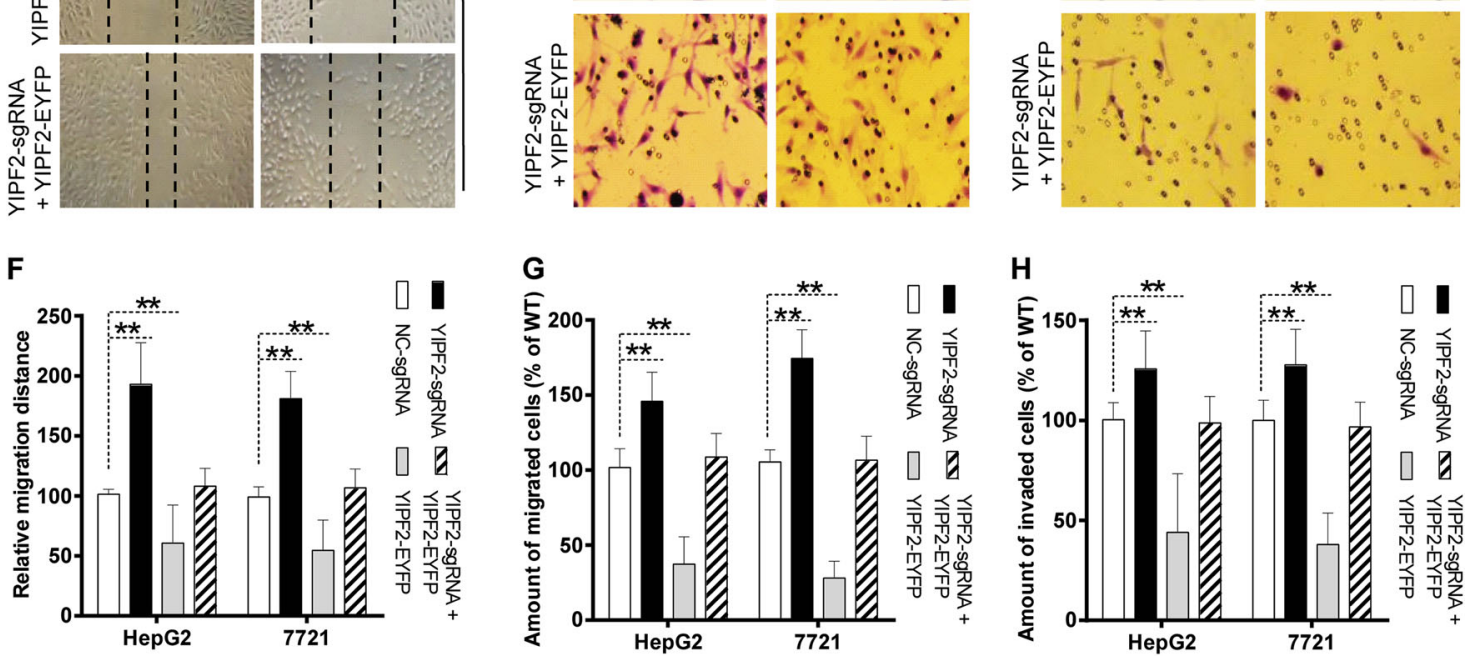

Fig. 8 YIPF2 knock-down enhanced the adhesion, motility, migration, and the invasion potential of HCC cells. YIPF2-KD HepG2 and 7721 cells were respectively transfected with the YIPF2/pEYFP plasmid. a Cell adhesion. Forty-eight hours after transfection, cells were re-seeded and equal numbers of cells were added to Matrigel-coated wells for $1 \mathrm{~h}$. Attached cells were crystal-violet-stained, lysed, and detected. $\mathbf{b}$ Cell proliferation. Sixteen hours after transfection, cells were re-seeded and equal numbers of cells were cultured for $32 \mathrm{~h}$. Numbers of viable cells were measured using the CCK-8 kit. c Cell motility. Cells were cultured until confluent. After scratching, cells were cultured with medium containing $10 \%$ FBS.

Photomicrographs at 10x magnifications were taken at $24 \mathrm{~h}$. $\mathbf{d}$ Cell migration. Twenty-four hours after transfection, cells were re-seeded and equal numbers of cells were added into the upper chamber. Twenty-four hours later, the cells migrating through the filter were crystal-violet-stained and counted. e Cell invasion. Similar procedures were performed as described in (d) except for adding cells into Matrigel-coated upper chamber and supplying HGF in the low chamber. Representative results from three independent experiments are shown in (c-e), and corresponding quantitative data were summarized in $(\mathbf{f}-\mathbf{h})$. Statis-tically significant differences compared with NC groups are shown: $n=3,{ }^{* *} p<0.01,{ }^{*} p<0.05$. WT: non-treated cells. NC-sgRNA: negative control 


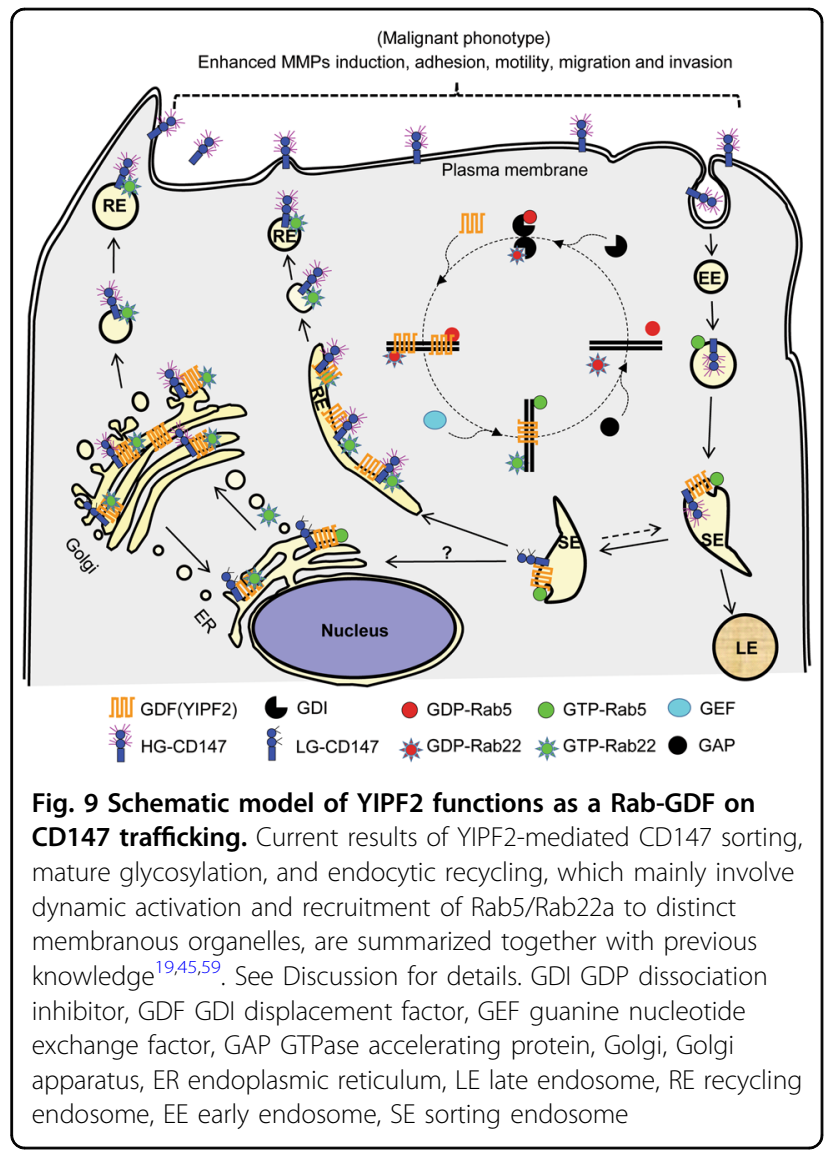

Rab5/Rab22a/pECFP-C1 plasmids were respectively transfected in HCC cells for gene overexpression.

Uptake measurement and ER/Golgi co-localization analysis

CD147 uptake and binding were measured as previously described $^{48-51}$. Incubation conditions for the $\mathrm{H} 18 \mathrm{Ab}$ complex were optimized (H18Ab, 1:500, anti-mouse AF488, 1:1000, $15 \mathrm{~min}$ ) in HCC cells. YIPF2-interference cells were PFA-fixed, triton-permeabilized, and stained by anti-KDEL/TGN38/GM130 Abs (1:100) together with anti-CD147 pcAb (1:2000) or with anti-GFP Ab (1:500, recognized Rab5/22a/pECFP), then stained by corresponding fluorescence-labeled $2^{\text {nd }} \mathrm{Ab}$. Images were analyzed with Image $J$ software, and co-localization was calculated as the Rank Weighted Coefficient $(\mathrm{RWC})^{52}$. In confocal imaging process, 60 cells were observed in each transfection. In flow cytometry measurements, 10,000 cells were counted per sample.

\section{Cell surface biotinylation and antigen recycling assay}

CD147 recycling was determined as previously described $^{45,53,54}$. Briefly, cells were labeled with biotin $(0.2 \mathrm{mg} /$ $\mathrm{ml}$ ) at $4{ }^{\circ} \mathrm{C}$, washed with glycine $(0.1 \mathrm{M}, \mathrm{PH} 8.0)$, and transferred for uptake at $37^{\circ} \mathrm{C}$. At $15 \mathrm{~min}$, cells were washed with PBS, and biotin was removed from cell- surface by incubation with $50 \mathrm{mM}$ DTT. After continueincubating at $37^{\circ} \mathrm{C}$ for $15 \mathrm{~min}$, cells were returned to the ice and biotin was removed from recycled proteins by a second DTT reduction. Cells were RIPA-lysed or further fractioned by utilizing cell fraction kits. Supernatants were corrected to equivalent protein concentration and levels of biotinylated CD147 (cell-surface portion vs intracellular non-recycled portion) were determined by co-IP.

\section{Rab GTPase activation assay}

Rab GTPase activation were performed as previously described $^{55,56}$. GST-EEA1 (residues 36-91) immobilized beads (for Rab22a activation) and GST-Rabaptin-5 (residues 739-e) immobilized beads (for Rab5 activation) were produced, respectively. After subjecting the collected pellets to SDS-PAGE, the GTP-bound Rab5 and Rab22a in samples were determined by Western blotting.

\section{Cell adhesion, proliferation, migration, motility, and invasion assays}

The processes were performed as previously described $^{10,57}$. In Transwell motility and invasion assays, the lower chamber was filled with 10\% FBS medium containing HGF $(20 \mathrm{ng} / \mathrm{ml})$.

\section{Cell fractionation, Western blot, co-IP, and Gelatin zymography assays}

The process was similar to that as previously descri$\operatorname{bed}^{10,57}$. Concentrated supernant from single-cultures were loaded for Gelatin zymography assay.

\section{Bioinformatics and statistical analysis}

Microarray mining analysis were performed based on GEPIA (Gene Expression Profiling Interactive Analysis) database (http://gepia.cancer-pku.cn//) ${ }^{58}$. The ANOVA differential method was used for tumor $(\mathrm{T})$ vs paired normal (N) samples. Confocal microscopy, flow cytometry, and Western blotting data were derived from three independent experiments. All data drawn from the experiments were analyzed using GraphPad Prism 5 software and are described as the mean \pm SEM values.

\section{Acknowledgements}

This work was supported by the National Natural Science Foundation of China (No. 81373318, 30700829), Natural Science Foundation of Tianjin (No.

13JCYBJC21000, 16JCYBJC23900) and Fundamental Research Funds for the Central Universities, Nankai University (No. 63191172). We are grateful to Prof. Byung-Ha Oh (Korea Advanced Institute of Science and Technology) for supplying EEA1, Rabaptin-5, Rab5 and Rab22a plasmids, Prof. Vytaute

Starkuviene (Heidelberg University) for supplying the YIPF2, YIPF3, YIPF4 plasmids, and Prof. T Kitamura (Tokyo University) for supplying the Plat-E cells.

\section{Authors' contributions}

S.H.Z. initiated the idea and the working approaches, supervised the research, and wrote the manuscript. S.S.Q. performed most of the experiments and data organization. L.J.S. and J.L. performed the cell fractionation, Western blot, and co-IP assays. P.Z. constructed the retroviral MSP-CDNA library. X.R.N. and Q.Z. performed the cell migration, motility, and invasion assays. G.H.J. and X.X.W. 
performed the gelatin zymography, cell adhesion, and proliferation experiments. J.T. supplied help in MAPPIT screening. J.L.J. and Z.N.C. performed the bioinformatics analysis. All authors read and approved the final manuscript.

\begin{abstract}
Author details
'Department of Cell Biology, School of Medicine, Nankai University, Tianjin, China. ${ }^{2}$ Department of Neurobiology, College of Life and Health Sciences, Northeastern University, Shenyang, China. ${ }^{3}$ Department of Clinical Laboratory, Cancer Hospital of Tianjin Medical University, Tianjin, China. ${ }^{4}$ Cytokine Receptor Laboratory, VIB Medical Biotechnology Center, Ghent University, Ghent, Belgium. ${ }^{5}$ Department of Cell Biology, National Translational Science Center for Molecular Medicine, State Key Laboratory of Cancer Biology, Fourth Military Medical University, Xi'an, China
\end{abstract}

\section{Conflict of interest}

The authors declare that they have no conflict of interest.

\section{Publisher's note}

Springer Nature remains neutral with regard to jurisdictional claims in published maps and institutional affiliations.

Supplementary Information accompanies this paper at (https://doi.org/ 10.1038/s41419-019-1709-8).

Received: 21 January 2019 Revised: 24 April 2019 Accepted: 27 May 2019 Published online: 12 June 2019

\section{References}

1. Goldenring, J. R. A central role for vesicle trafficking in epithelial neoplasia: intracellular highways to carcinogenesis. Nat. Rev. Cancer 13, 813-820 (2013).

2. Eaton, S. \& Martin-Belmonte, F. Cargo sorting in the endocytic pathway: a key regulator of cell polarity and tissue dynamics. Cold Spring Harb. Perspect. Biol. $\mathbf{6}$ a016899 (2014).

3. Sorkin, A. \& von Zastrow, M. Endocytosis and signalling: intertwining molecular networks. Nat. Rev. Mol. Cell Biol. 10, 609-622 (2009).

4. Sigismund, S. et al. Endocytosis and signaling: cell logistics shape the eukaryotic cell plan. Physiol. Rev. 92, 273-366 (2012).

5. Yan, L., Zucker, S. \& Toole, B. P. Roles of the multifunctional glycoprotein, emmprin (basigin; CD147), in tumour progression. Thromb. Haemost. 93 199-204 (2005).

6. Bai, Y., Huang, W., Ma, L. T., Jiang, J. L. \& Chen, Z. N. Importance of Nglycosylation on CD147 for its biological functions. Int. J. Mol. Sci. 15, 6356-6377 (2014)

7. Grass, G. \& Toole, B. How, with whom and when: an overview of CD147mediated regulatory networks influencing matrix metalloproteinase activity. Biosci. Rep. 36, e00283 (2015).

8. Muramatsu, T. Basigin (CD147), a multifunctional transmembrane glycoprotein with various binding partners. J. Biochem. 159, 481-490 (2016).

9. Huang, Q. et al. CD147 promotes reprogramming of glucose metabolism and cell proliferation in HCC cells by inhibiting the p53-dependent signaling pathway. J. Hepatol. 61, 859-866 (2014).

10. Zhao, P. et al. HAb18G/CD147 promotes cell motility by regulating annexin IIactivated RhoA and Rac1 signaling pathways in hepatocellular carcinoma cells. Hepatology 54, 2012-2024 (2011).

11. Wu, B. et al. Basigin-mediated redistribution of CD98 promotes cell spreading and tumorigenicity in hepatocellular carcinoma. J. Exp. Clin. Cancer Research 34, 110 (2015).

12. Weigert, R., Yeung, A. C., Li, J. \& Donaldson, J. G. Rab22a regulates the recycling of membrane proteins internalized independently of clathrin. Mol Biol. Cell 15, 3758-3770 (2004).

13. Kauppi, M. et al. The small GTPase Rab22 interacts with EEA1 and controls endosomal membrane trafficking. J. Cell Sci. 115, 899-911 (2002).

14. Zhu, H., Liang, Z. \& Li, G. Rabex-5 is a Rab22 effector and mediates a Rab22Rab5 signaling cascade in endocytosis. Mol. Biol. Cell 20, 4720-4729 (2009).

15. Eyster, C. A. et al. Discovery of new cargo proteins that enter cells through clathrin-independent endocytosis. Traffic 10, 590-599 (2009).
16. Radhakrishna, H. \& Donaldson, J. G. ADP-ribosylation factor 6 regulates a novel plasma membrane recycling pathway. J. Cell Biol. 139, 49-61 (1997).

17. Maldonado-Baez, L., Cole, N. B., Kramer, H. \& Donaldson, J. G. Microtubuledependent endosomal sorting of clathrin-independent cargo by Hook1. J. Cell Biol. 201, 233-247 (2013).

18. Grant, B. D. \& Donaldson, J. G. Pathways and mechanisms of endocytic recycling. Nat. Rev. Mol. Cell Biol. 10, 597-608 (2009).

19. Tang, W., Chang, S. \& Hemler, M. Links between CD147 function, glycosylation, and caveolin-1. Mol. Biol. Cell 15, 4043-4050 (2004).

20. Tang, W. \& Hemler, M. E. Caveolin-1 regulates matrix metalloproteinases-1 induction and CD147/EMMPRIN cell surface clustering. J. Biol. Chem. 279, 11112-11118 (2004).

21. Gallagher, S. M., Castorino, J. J., Wang, D. \& Philp, N. J. Monocarboxylate transporter 4 regulates maturation and trafficking of CD147 to the plasma membrane in the metastatic breast cancer cell line MDA-MB-231. Cancer Res. 67, 4182-4189 (2007)

22. Doherty, J. R. \& Cleveland, J. L. Targeting lactate metabolism for cancer therapeutics. J. Clin. Investig. 123, 3685-3692 (2013).

23. Pushkarsky, T. et al. Cell surface expression of CD147/EMMPRIN is regulated by cyclophilin 60. J. Biol. Chem. 280, 27866-27871 (2005).

24. Yurchenko, V. et al. Regulation of CD147 cell surface expression: involvement of the proline residue in the CD147 transmembrane domain. J. Biol. Chem. 280, 17013-17019 (2005).

25. Mauris, J., Woodward, A. M., Cao, Z., Panjwani, N. \& Arqueso, P. Molecular basis for MMP9 induction and disruption of epithelial cell-cell contacts by galectin-3. J. Cell Sci. 127, 3141-3148 (2014).

26. Gerke, V., Creutz, C. E. \& Moss, S. E. Annexins: linking Ca2 + signalling to membrane dynamics. Nat. Rev. Mol. Cell Biol. 6, 449-461 (2005).

27. Zhang, W. et al. Annexin A2 promotes the migration and invasion of human hepatocellular carcinoma cells in vitro by regulating the shedding of CD147harboring microvesicles from tumor cells. PLOS ONE 8, e67268 (2013).

28. Eyckerman, $\mathrm{S}$. et al. Design and use of a mammalian protein-protein interaction trap (MAPPIT). Sci. STKE2002, pl18 (2002).

29. Suda, Y., Kurokawa, K. \& Nakano, A. Regulation of ER-Golgi transportdynamics by GTPases in budding yeast. Front. Cell Dev. Biol. 5, 122 (2017).

30. Wandinger-Ness, A. \& Zerial, M. Rab proteins and the compartmentalization of the endosomal system. Cold Spring Harb. Perspect. Biol. 6, a022616 (2014).

31. Numrich, J. \& Ungermann, C. Endocytic Rabs in membrane trafficking and signaling. Biol. Chem. 395, 327-333 (2014).

32. Brauer, P. R. MMPs-role in cardiovascular development and disease. Front. Biosci. 11, 447-478 (2006).

33. Maldonado-Baez, L. \& Donaldson, J. G. Hook1, microtubules, and Rab22 mediators of selective sorting of clathrin-independent endocytic cargo proteins on endosomes. Bioarchitecture 3, 141-146 (2013).

34. Saraste, J. Spatial and Functional Aspects of ER-Golgi Rabs and Tethers. Front. Cell Dev. Biol. 4, 28 (2016)

35. Zhou, S., Zhou, H., Walian, P. J. \& Jap, B. K. CD147 is a regulatory subunit of the gamma-secretase complex in Alzheimer's disease amyloid beta-peptide production. Proc. Natl Acad. Sci. USA 102, 7499-7504 (2005).

36. Nahalkova, J. et al. CD147, a gamma-secretase associated protein is upregulated in Alzheimer's disease brain and its cellular trafficking is affected by presenilin-2. Neurochem. Int. 56, 67-76 (2010).

37. Eyckerman, S. et al. Design and application of a cytokine-receptor-based interaction trap. Nat. Cell Biol. 3, 1114-1119 (2001).

38. Shakoori, A. et al. Identification of a five-pass transmembrane protein family localizing in the Golgi apparatus and the ER. Biochem. Biophys. Res. Commun 312, 850-857 (2003).

39. Kranjc, T. et al. Functional characterisation of the YIPF protein family in mammalian cells. Histochem. Cell Biol. 147, 439-451 (2017).

40. Barrowman, J., Wang, W., Zhang, Y. \& Ferro-Novick, S. The Yip1 p.Yif1 p complex is required for the fusion competence of endoplasmic reticulum-derived vesicles. J. Biol. Chem. 278, 19878-19884 (2003).

41. Soonthornsit, J. et al. YIPF1, YIPF2, and YIPF6 are medial-/trans-Golgi and trans-Golgi network-localized Yip domain family proteins, which play a role in the Golgi reassembly and glycan synthesis. Exp. Cell Res. 353, 100-108 (2017).

42. Simpson, J. C. et al. Genome-wide RNAi screening identifies human proteins with a regulatory function in the early secretory pathway. Nat. Cell Biol. 14, 764-774 (2012).

43. Heidtman, M., Chen, C. Z., Collins, R. N. \& Barlowe, C. Yos1p is a novel subunit of the Yip1p-Yif1p complex and is required for transport between the 
endoplasmic reticulum and the Golgi complex. Mol. Biol. Cell 16, 1673-1683 (2005).

44. Huang, W. et al. Modulation of CD147-induced matrix metalloproteinase activity: role of CD147 N-glycosylation. Biochem. J. 449, 437-448 (2013).

45. Dutta, D. \& Donaldson, J. G. Sorting of Clathrin-Independent Cargo Proteins Depends on Rab35 Delivered by Clathrin-Mediated Endocytosis. Traffic $\mathbf{1 6}$ 994-1009 (2015).

46. Calero, M., Winand, N. J. \& Collins, R. N. Identification of the novel proteins Yip4p and Yip5p as Rab GTPase interacting factors. FEBS Lett. 515, 89-98 (2002).

47. Zhou, Y. et al. Rab22a enhances CD147 recycling and is required for lung cancer cell migration and invasion. Exp. Cell Res. 357, 9-16 (2017).

48. Niu, X. et al. GRP75 modulates oncogenic Dbl-driven endocytosis derailed via the CHIP-mediated ubiquitin degradation pathway. Cell Death Dis. 9, 971 (2018).

49. Chen, H. et al. GRP75 upregulates clathrin-independent endocytosis through actin cytoskeleton reorganization mediated by the concurrent activation of Cdc42 and RhoA. Exp. Cell Res. 343, 223-236 (2016).

50. Gao, Z. et al. Mitochondria chaperone GRP75 moonlighting as a cell cycle controller to derail endocytosis provides an opportunity for nanomicrosphere intracellular delivery. Oncotarget 8, 58536-58552 (2017).
51. Niu, X. et al. Macropinocytosis activated by oncogenic Dbl enables specific targeted delivery of Tat/pDNA nano-complexes into ovarian cancer cells. Int. J. Nanomed. 13, 4895-4911 (2018).

52. Singan, V. R., Jones, T. R., Curran, K. M. \& Simpson, J. C. Dual channel rank-based intensity weighting for quantitative co-localization of microscopy images. BMC Bioinform. 12, 407 (2011).

53. Bretscher, M. S. \& Lutter, R. A new method for detecting endocytosed proteins. EMBO J. 7, 4087-4092 (1988).

54. Schmid, S. L. \& Smythe, E. Stage-specific assays for coated pit formation and coated vesicle budding in vitro. J. Cell Biol. 114, 869-880 (1991).

55. Ku, B. et al. VipD of Legionella pneumophila targets activated Rab5 and Rab22 to interfere with endosomal trafficking in macrophages. PLOS Pathogens 8 , e1003082 (2012).

56. Sivars, U., Aivazian, D. \& Pfeffer, S. R. Yip3 catalyses the dissociation of endosomal Rab-GDI complexes. Nature 425, 856-859 (2003).

57. Zhao, P. et al. Annexin II promotes invasion and migration of human hepatocellular carcinoma cells in vitro via its interaction with HAb18G/CD147. Cancer Sci. 101, 387-395 (2010).

58. Tang, Z. et al. GEPIA: a web server for cancer and normal gene expression profiling and interactive analyses. Nucleic Acids Res. 45, W98-W102 (2017).

59. Barrowman, J. \& Novick, P. Three Yips for Rab recruitment. Nat. Cell Biol. $\mathbf{5}$ 955-956 (2003). 